PROCEEDINGS OF THE AMERICAN MATHEMATICAL SOCIETY

Volume 124, Number 8, August 1996

\title{
CARTAN INVARIANTS OF GROUP ALGEBRAS OF FINITE GROUPS
}

\author{
SHIGEO KOSHITANI \\ (Communicated by Ronald M. Solomon) \\ Dedicated to Professor Takeshi Kondo on his 60th birthday
}

\begin{abstract}
We give a result on Cartan invariants of the group algebra $k G$ of a finite group $G$ over an algebraically closed field $k$, which implies that if the Loewy length (socle length) of the projective indecomposable $k G$-module corresponding to the trivial $k G$-module is four, then $k$ has characteristic 2 . The proof is independent of the classification of finite simple groups.
\end{abstract}

\section{INTRODUCTION AND NOTATION}

Let $k G$ be the group algebra of a finite group $G$ over a field $k$ of characteristic $p>0$. By a $k G$-module we always mean a right $k G$-module. In this paper we discuss Cartan invariants of $k G$, especially those of the projective cover $P=P\left(k_{G}\right)$ of the trivial $k G$-module $k_{G}$. Let $j$ be the Loewy length of $P$, that is, $j$ is the least positive integer $t$ such that $P J^{t}=0$ where $J$ is the Jacobson radical of $k G$. It is well-known that the structure of $G$ is completely determined provided $j \leqslant 2$ by Maschke and Wallace [8]. The structure of $G$ with $j=3$ has not been determined yet. There is, however, a result by Okuyama [7, Theorem 2]. He proved that Sylow 2-subgroups of $G$ are dihedral if $j=3$ under the condition $p=2$. Here we investigate finite groups $G$ satisfying $j=4$. As a matter of fact, the condition $j=4$ seems stronger and more mysterious than the condition $j=3$ as seen below.

Namely, our results of this note are the following.

Theorem. Let $k G$ be the group algebra of a finite group $G$ over an algebraically closed field $k$ of characteristic $p>0$. Assume that $S$ is a simple $k G$-module which is self-dual, that is, the dual module $S^{*}=\operatorname{Hom}_{k}(S, k)$ of $S$ is isomorphic to $S$ itself as $k G$-modules. If $p$ is odd, then there is a simple $k G$-module $T$ such that $T$ is self-dual and the Cartan invariant $c(S, T)$ with respect to $S$ and $T$ is odd.

Corollary. Let $k$ be an arbitrary field and $G$ a finite group. If the Loewy length of the projective indecomposable $k G$-module corresponding to the trivial $k G$-module is four, then $k$ has characteristic 2.

Received by the editors August 17, 1994 and, in revised form, February 7, 1995.

1991 Mathematics Subject Classification. Primary 20C05, 20C20.

Supported in part by the Alexander von Humboldt Foundation, the Mathematical Prizes Fund, the University of Oxford and the Sasakawa Foundation. 
Note that the theorem and the corollary do not depend on the classification of finite simple groups.

Remark. It should be remarked that if $G$ is the symmetric group on 4 letters and if $k$ has characteristic 2 , then the Loewy length of the projective indecomposable $k G$ module corresponding to the trivial $k G$-module is four. The situation for $p$-solvable groups is treated completely in our previous paper [2].

Throughout this paper we use the following notation and terminology. By a $k G$-module we mean a finitely generated right $k G$-module. We write $k_{G}$ for the trivial $k G$-module, and $P\left(k_{G}\right)$ for its projective cover. We denote by $J(k G)$ the Jacobson radical of $k G$. Let $M$ be a $k G$-module. Then $j(M)$ denotes the Loewy length of $M$, namely, $j(M)$ is the least positive integer $j$ such that $M \cdot J(k G)^{j}=0$, and $\operatorname{Soc}(M)$ denotes the socle of $M$ (see [3, I $\S 8]$ ). We write $M^{*}$ for the dual module of $M$, that is, $M^{*}=\operatorname{Hom}_{k}(M, k)$ and this is also a right $k G$-module (see [3, I $\left.\S 6\right]$ ). Then, $M$ is called self-dual if $M \simeq M^{*}$ as $k G$-modules. For $k G$-modules $M$ and $N$, we denote $\operatorname{dim}_{k}\left[\operatorname{Hom}_{k G}(M, N)\right]$ by $[M, N]^{G}$. For simple $k G$-modules $S$ and $T$, $c(S, T)$ denotes the Cartan invariant with respect to $S$ and $T$ (usually, a notation $c_{S, T}$ is used instead). Other notation and terminology follow the books of Feit [1] and Landrock [3].

\section{Calculation of determinants}

In this section we give several lemmas on elementary computation of determinants for matrices over a field of characteristic 2. Throughout this section all entries of matrices are elements in a field of characteristic 2. This assumption is essential here.

Lemma 1.1. For non-negative integers $m$ and $n$, let $A$ be a square matrix of size $m+2 n+2$ of the form

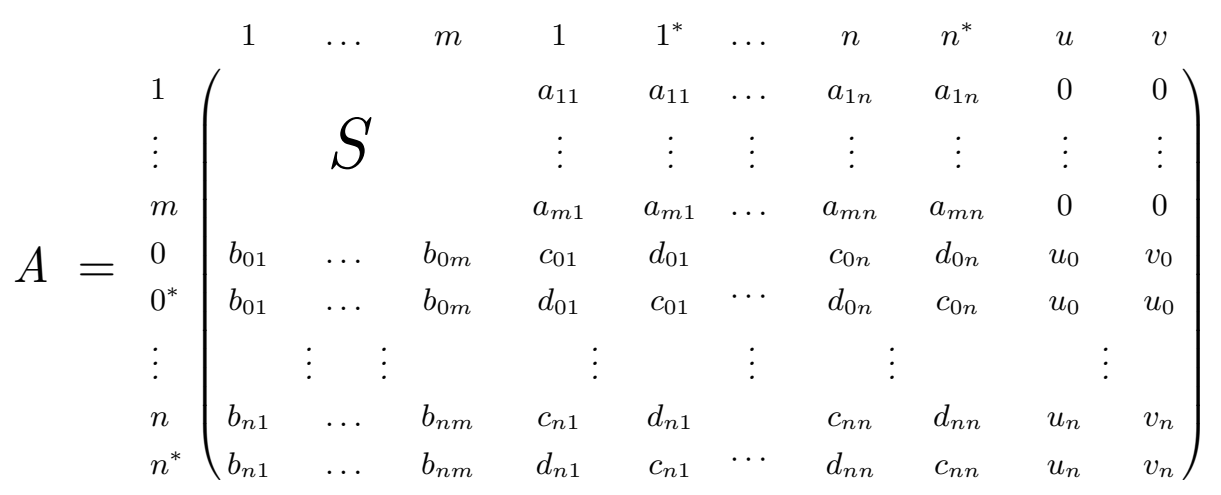

where $S$ is an $(m \times m)$-matrix. Then, $\operatorname{det} A=0$.

Proof. We prove the lemma by induction on $n$. If $n=0$, then $\operatorname{det} A=0$ since two rows indexed by 0 and $0^{*}$ are the same. Assume $n \geqslant 1$. Expand $A$ with respect to the last column indexed by $v$. Then, by symmetry, it is enough to show that the 
determinant of the following square matrix $B$ of size $m+2 n+1$ is zero:

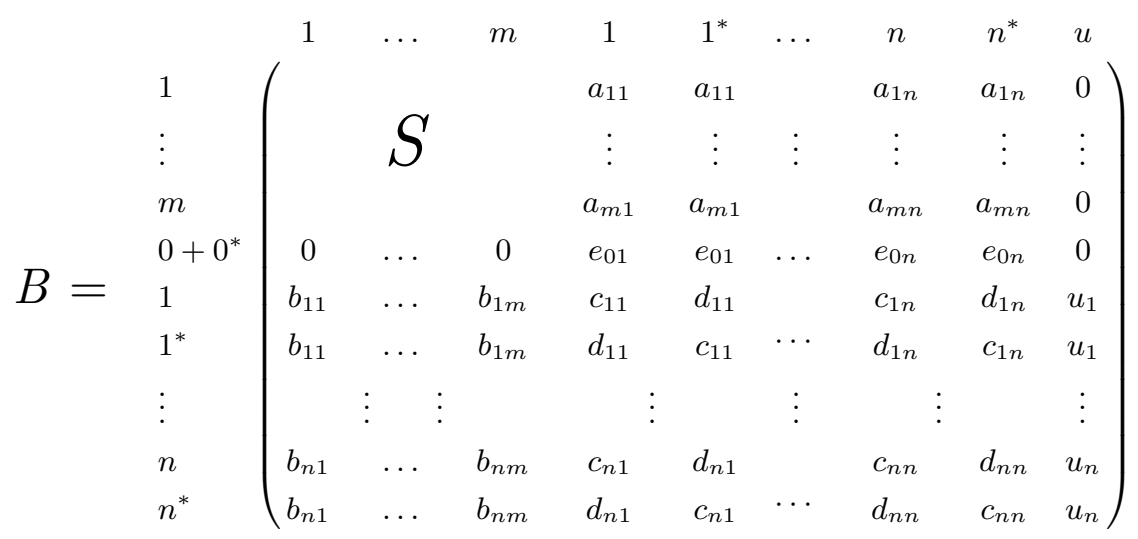

where $e_{0 i}=c_{0 i}+d_{0 i}$ for $i=1, \ldots, n$. Now, expand $B$ with respect to the final column indexed by $u$. So, again by symmetry, it suffices to prove $\operatorname{det} C=0$ for the following square matrix $C$ of size $m+2 n$ :

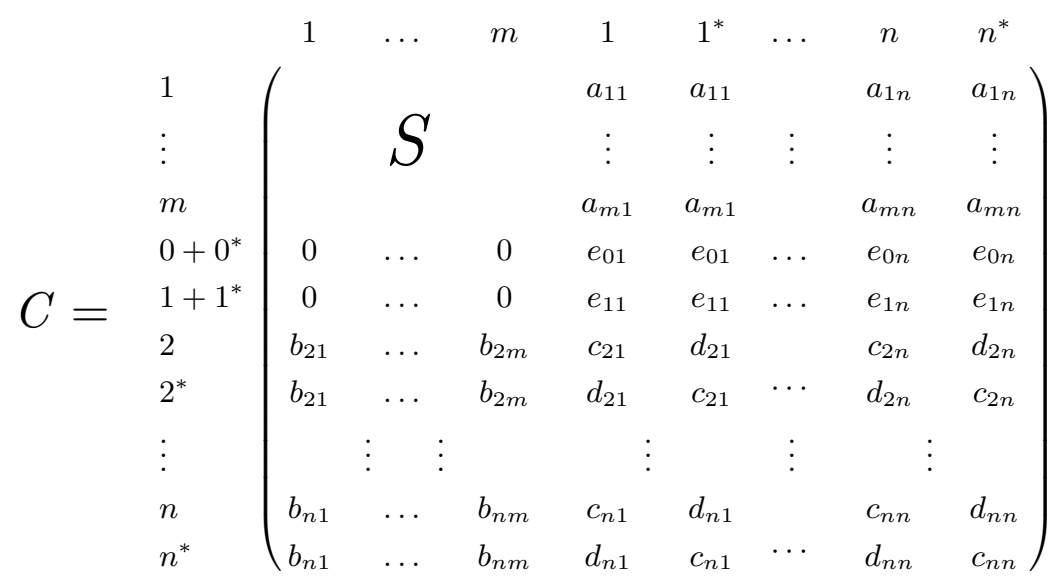

where $e_{1 i}=c_{1 i}+d_{1 i}$ for $i=1, \ldots, n$. Then, by carrying two rows indexed by $0+0^{*}$ and $1+1^{*}$ to the end, and then by taking its transpose, we have $\operatorname{det} C=0$ by induction.

Lemma 1.2. For non-negative integers $m$ and $n$, let $A$ be a square matrix of size $m+2 n+1$ of the form

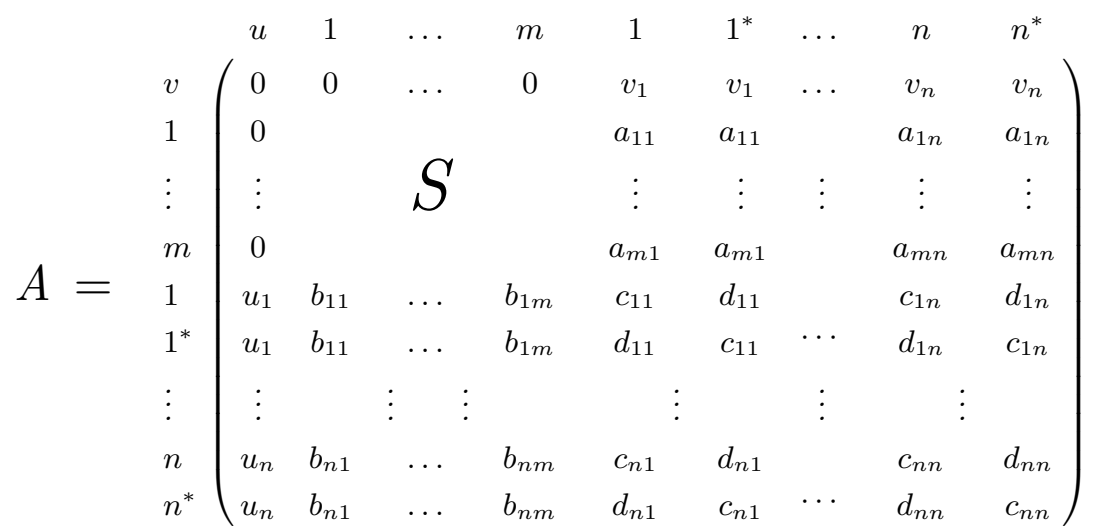

where $S$ is an $(m \times m)$-matrix. Then, $\operatorname{det} A=0$. 
Proof. First of all, expand $A$ with respect to the first row indexed by $v$, and sum each pair of determinants of size $m+2 n$ that have the same coefficient $v_{i}$ for $i=1, \ldots, n$. Namely, we can write $\operatorname{det} A=\sum_{i=1}^{n} v_{i} \cdot \operatorname{det} B_{i}$, where each $B_{i}$ is a square matrix of size $m+2 n$ and each $B_{i}$ has the same form as in Lemma 1.1 by a suitable exchanging of columns. Therefore, $\operatorname{det} A=0$ by Lemma 1.1.

\section{LEMMAS}

In this section we state several lemmas which will be used in the proofs of our results. Throughout this section we assume that $k$ is an algebraically closed field of characteristic $p>0$, and we fix a finite group $G$ such that $p$ divides the order of G.

Lemma 2.1 (Webb [9, Theorem E]). Let $P=P\left(k_{G}\right)$ and assume $j(P) \geqslant 3$. If $p$ is odd, then $P \cdot J(k G) / \operatorname{Soc}(P)$ is an indecomposable $k G$-module.

Lemma 2.2 ([6, Lemma 1.2]). If $M$ is an indecomposable $k G$-module with $j(M)=$ 2 , then $M \cdot J(k G)=\operatorname{Soc}(M)$.

Lemma 2.3 ([3, II Corollary 6.9]). For $k G$-modules $M$ and $N$,

$$
[M, N]^{G}=\left[N^{*}, M^{*}\right]^{G} .
$$

Lemma 2.4 ([3, I Lemma 8.4 (i)]). For a $k G$-module $M$,

$$
[M /(M \cdot J(k G))]^{*} \simeq \operatorname{Soc}\left(M^{*}\right) \quad \text { as } k G \text {-modules. }
$$

Lemma 2.5 (Landrock). For simple $k G$-modules $S$ and $T$,

$$
c(S, T)=c(T, S)=c\left(S^{*}, T^{*}\right)=c\left(T^{*}, S^{*}\right) .
$$

Proof. We get the assertion from [1, I Lemma 14.9 and Theorem 16.7] and Landrock's result [4, Theorem A] (cf. [3, I Theorem 9.9]).

\section{Proofs}

In this section we give proofs of the theorem and the corollary in the introduction.

Proof of Theorem. Let $B$ be a block ideal of $k G$ containing $S$. Since $S$ is self-dual, $Y^{*}$ is a simple $k G$-module in $B$ again if $Y$ is a simple $k G$-module in $B$ (see [3, I Proposition 10.8]). Thus, let $S_{0}=S, S_{1}, \ldots, S_{m}, T_{1}, T_{1}{ }^{*}, \ldots, T_{n}, T_{n}{ }^{*}$ all be nonisomorphic simple $k G$-modules in $B$; and $S_{i} \simeq S_{i}{ }^{*}$ for all $i$ and $T_{j} \not T_{j}{ }^{*}$ for all $j$. We denote by $C$ the Cartan matrix for $B$, and let $\bar{C}$ be its image induced by the canonical epimorphism $\mathbb{Z} \rightarrow \mathbb{Z} / 2 \mathbb{Z}$.

Now, suppose that $c\left(S_{0}, S_{i}\right)$ is even for all $i=0, \ldots, m$. Then Lemma 2.5 implies that $\bar{C}$ has the same form as in Lemma 1.2, so that $\operatorname{det} \bar{C}=0$ from Lemma 1.2. This means $\operatorname{det} C$ is even, which contradicts Brauer's result [1, IV Theorem 3.9]).

Proof of Corollary. First of all, we may assume that $k$ is algebraically closed (see [5, Proposition 12.11]). Let $J=J(k G), P=P\left(k_{G}\right)$ and $M=P J / \operatorname{Soc}(P)$.

Assume $p$ is odd. By Lemmas 2.1 and 2.2, $M$ is an indecomposable $k G$-module with $M J=\operatorname{Soc}(M)$. Then, the Theorem implies that there is a simple $k G$-module $T$ such that $T$ is self-dual and $c\left(k_{G}, T\right)$ is odd. On the other hand, since $T$ and $M$ are both self-dual, and since $M J=\operatorname{Soc}(M)$, it follows from Lemmas 2.3 and 2.4 that

$$
[M / M J, T]^{G}=\left[T,(M / M J)^{*}\right]^{G}=[T, \operatorname{Soc}(M)]^{G}=[T, M J]^{G},
$$


which says that the multiplicities of $T$ in $M / M J$ and $M J$ as direct summands are the same, so that $c\left(k_{G}, T\right)$ is even, a contradiction.

\section{ACKNOWLEDGEMENTS}

The author is so grateful to the referee for her or his nice and kind advice, which has improved the main result in the first version of the paper.

A part of this work was done while the author was staying at the Institute for Experimental Mathematics, Essen University (July - October 1992), and at the Mathematical Institute, University of Oxford (September 1992). He would like to thank the Humboldt Foundation, the Mathematical Prizes Fund, the University of Oxford and the Sasakawa Foundation for their financial support, and also he would

like to express his great thanks to Professor G.O. Michler and the people around him, and Professor K. Erdmann for their hospitality.

\section{REFERENCES}

[1] W. Feit, The representation theory of finite groups, North-Holland, Amsterdam, 1982. MR 83g:20001

[2] S. Koshitani, Group algebras of finite p-solvable groups with radicals of the fourth power zero, Proc. Royal Soc. Edinburgh 92A (1982), 205-231. MR 84b:2007

[3] P. Landrock, Finite group algebras and their modules, London Math. Soc. Lecture Note Series Vol.84, Cambridge, 1983. MR 85h:20002

[4] P. Landrock, The Cartan matrix of a group algebra modulo any power of its radical, Proc. Amer. Math. Soc. 88 (1983), 205-206. MR 84f:20010

[5] G.O. Michler, Blocks and centers of group algebras, in Lectures on rings and modules, Springer Lecture Notes in Math. Vol.246, Springer, Berlin, 1972, pp.429-563. MR 48:11274

[6] W. Müller, Unzerlegbare Moduln über artinschen Ringen, Math. Z. 137 (1974), 197-226. MR 50:4665

[7] T. Okuyama, On blocks of finite groups with radical cube zero, Osaka J. Math. 23 (1986), 461-465. MR 87k:20024

[8] D.A.R. Wallace, Group algebras with radicals of square zero, Proc. Glasgow Math. Assoc. 5 (1962), 158-159. MR 25:3986

[9] P.J. Webb, The Auslander-Reiten quiver of a finite group, Math. Z. 179 (1982), 97-121. MR 83b:16029

Department of Mathematics, Faculty of Science, Chiba University, Yayoi-cho, ChibaCITY, 263, JAPAN

E-mail address: koshitan@math.s.chiba-u.ac.jp 